\title{
Los nuevos aprendizajes del sujeto digital
}

\section{Aras, Roberto E.}

Resumen:

Si tuviéramos que trazar rápidamente la evolución de los intercambios entre las tecnologías de la información y la educación deberíamos distinguir, al menos, tres fases: las experiencias pioneras, la integración, y la instrumentalización de recursos digitales y virtualización. Sin embargo, ese proceso de apropiación ha sido también de maduración y, por ende, ha motivado una reflexión sobre las propias prácticas docentes, señalando lí- mites y posibilidades. Quizás, hoy asistimos al ingreso en otra etapa de aquella trayectoria evolutiva que se podría denominar como de "postdigitalización". Aquí aparecen, entonces, cuestiones que desafían no

Cuadernos del Centro de Estudios de Diseño y Comunicación N N 64

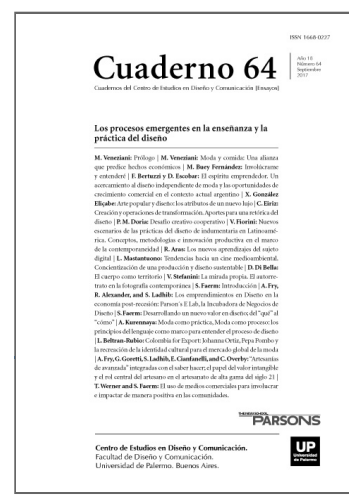

ISSN: 1668-0227

Los procesos

emergentes en la

enseñanza y la

práctica del diseño

Año XVIII, Septiembre 2017, Buenos Aires, Argentina | 326 páginas

descargar PDF ver índice de la publicación

Ver todos los libros de la publicación

compartir en Facebook

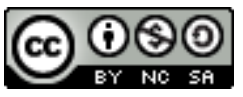
Esta obra está bajo una Licencia Creative Commons Atribución-NoComercialCompartirlgual 4.0 Internacional sólo la manera de educar (abarcativa de la didáctica de los saberes) sino que también interpelan la formación de nuevos hábitos culturales cuyo ejercicio no reconoce mediatizaciones, elimina instancias de control y, muchas veces, propone lógicas de "acción directa" detrás de las que se oculta un poder que necesita ser reconocido y medido por sus consecuencias. La magnitud de este cambio sucedido en el seno de la "sociedad del Conocimiento" dispara un alerta educativo que solicita nuestra atención sobre los criterios de organización del naciente "Currículo de intervención digital"; una intervención que se ha extendido -hace tiempo ya- desde los objetos hasta las personas. Por todo ello, la relación con los dispositivos, la selección de plataformas, la disponibilidad de la información, la ubicuidad de las consultas, la intervención y administración de redes, y el desarrollo de aplicaciones constituyen el novedoso ambiente digital que requiere abordajes transdisciplinarios y una mirada humanizadora para no sucumbir al peligro de generar una masificación de los sujetos adaptada a los paradigmas del siglo XXI.

Palabras clave:

digital - educación - redes - TIC'S - conocimiento - sujeto 
(*) Doctor en Filosofía (Universidad de Navarra, España). Profesor Titular Ordinario de Filosofía y Antropología, y Cuestiones Profesionales y Ética en la Facultad de Ciencias Sociales de la Pontificia Universidad Católica Argentina, donde también es Secretario Académico. Es miembro académico del Comité Latinoamericano de Acreditación de Educación en Periodismo (CLAEP) dependiente de la Sociedad Interamericana de Prensa y Presidente de la Fundación Ortega y Gasset de Argentina.

\section{Humanidad y cultura digital}

En los últimos veinticinco años la percepción de los avances en las tecnologías de la comunicación y de la información ha recorrido el amplio arco que va desde el optimismo sin matices hasta la crítica afectada por expresiones de prevención y sospecha. Un testimonio de las variaciones acontecidas en ese campo lo ofrece la bibliografía que ha intentado advertir sobre los tiempos cambiantes que asomaban desde la década del noventa. Así, los clásicos Being Digital [Ser Digital, 1995] de Nicholas Negroponte o The road ahead [Camino al futuro, 1995] de Bill Gates, destilan una visión positiva de los cambios que produciría en la humanidad la inserción cada vez mayor y más extendida de los recursos informáticos. Se trataba de libros pioneros que buscaban anticipar mediante una visión totalizadora la aurora de una época de renovada confianza en el ser humano y sus logros. Sin embargo, ya en el siglo XXI, títulos como The Social Life of Information [La vida social de la información, Prentice Hall, 2001] de John Seely Brown y Paul Duguid, publicado por Harvard Business School Press (2000), se atrevían a mirar un aspecto que todavía no alcanzaba a suscitar la atención de los sociólogos y de los analistas de la conducta: qué sucederá, con el paso del tiempo y la sinergia entre hombres y computadoras, con los hábitos intelectuales y sociales; qué podría esperarse de las relaciones "en línea" o de las maneras de relacionarse entre las personas, o entre las personas y objetos (dispositivos, plataformas) tan sofisticados que algunos denominan "inteligentes". Este nuevo ambiente digital comenzó a provocar dudas y a requerir abordajes transdisciplinarios que se reflejaron en la aparición de una serie de textos cuya misión parecía ser la de insertar en el ambiente categorías que parecían olvidadas por una cierta aproximación ingenua a los beneficios del fenómeno de la disponibilidad de la información masiva, de la ubicuidad de las consultas, de la intervención y administración de redes, y del desarrollo de aplicaciones capaces de regular hasta los mínimos aspectos de la vida. Si bien aparecieron voces disonantes en aquellos primeros estadios de la producción académica que acompañaba a los desarrollos informáticos (por ejemplo, el libro de Neil Postman, Technopoly: the surrender of culture to technology (Vintage Books, 1993) o el caso de Life on Screen (Simon \& Schuster, 1995) [La vida en la pantalla, Planeta, 2011] de la célebre Sherry Turkle quien, además de poseer un Doctorado en Sociología, había realizado estudios en Psicología de la Personalidad y, especialmente, trabajaba sobre la interacción entre los seres humanos y la tecnología a partir de una perspectiva psicoanalítica y piagetiana) no fue sino durante los últimos diez años que el resultado de ese movimiento reflexivo se consolidó en obras como The Shallows: What the Internet is doing to our brains (Norton \& Co., 2010) [Superficiales: ¿Que está haciendo Internet con nuestras mentes?, Taurus, 2011] de Nicholas G. Carr o Alone Together. Why we expect more from technology and less from each other (Basic Books, 2012) de la mencionada Turkle. Un caso curioso de esa evolución argumentativa lo presenta la obra de Howard Rheingold, The virtual community (MIT PRess, 1993) [Comunidades virtuales] que en el año 2000 es actualizada con un capítulo más, titulado "Disinformocracy", en el cual aparecen claramente las objeciones a la posible manipulación e influencia de los discursos mediatizados. En el mismo camino de denuncia aparecen los textos The Cult of the Amateur: How Today's Internet Is Killing Our Culture (Doubleday/Currency, 2007) y The internet is not the answer (Grove/Atlantic, 2015), ambos de Andrew Keen; The Net Delusion: The dark side of Internet freedom (Evgeny Morozov, Public Affairs, 2011) o You are not a 
gadget (Jaron Lanier, Penguin/ Random House, 2011). Sin embargo, frente a estas posiciones escépticas hay todavía quienes destacan que la era de las comunicaciones digitales y las redes sociales es altamente beneficiosa para el género humano. Ese es el punto de partida de Cognitive Surplus: Creativity and Generosity in a Connected Age (Penguin Press, 2010) de Clay Shirky. Ahora bien, de todo lo antedicho podría obtenerse una conclusión trivial afirmando que, desde que comenzó su existencia, la tecnología (no importa ahora el grado de su desarrollo) siempre ha sido objeto de una acusación de ambigüedad que alternaba entre una opinión positiva o una negativa según las consecuencias de su uso, objeción que cabe, en rigor, para todo producto humano técnico. Pero lo que intentaré demostrar a través de estas páginas es que la informática y los avances en las redes comunicacionales de las últimas dos décadas significan un cambio que no es comparable con otros momentos revolucionarios en la historia de las invenciones humanas, por cuanto involucran modificaciones en lo más íntimo del hombre: sus esquemas de pensamiento y de acción. En este sentido, se desprende que la educación es primariamente afectada por este cambio y que la manera en que se constituye la relación entre el maestro y el discípulo, de los maestros entre sí y de los discípulos entre sí, pasa por una etapa de necesaria reflexión. El primitivo encuentro que se produce entre el alumno y la tecnología en el aula (sucedáneo al que se verifica mucho antes en el hogar) genera hábitos perceptivos (dinámicas de visualización), motores (destrezas manuales) e intelectuales (nuevos conectores lógicos y sintácticos) que perdurarán en el tiempo y acompañarán los siguientes procesos de inserción social (redes, trabajos colaborativos, etc.). El aprendizaje mediado por instrumentos informáticos o intervenido desde las redes requiere de un "meta-aprendizaje" transversal que ilumine cuáles son los presupuestos de su utilización y, sobre todo, qué nuevas maneras de vivir nuestra humanidad nos están proponiendo. Como bien recuerda Keen (2015), Marshall McLuhan, empleando una metáfora que le presta un discurso de Churchill de 1944, establece que "damos forma a nuestras herramientas y luego nuestras herramientas nos forman a nosotros". Gran parte de este planteo subyace en la bibliografía que fue enumerada al comienzo y que da cuenta de la alta motivación de sus autores para desentrañar las fortalezas y debilidades del paradigma digital. Pero la deconstrucción de la propuesta habitual de las TIC's demanda una visión humanística y transdisciplinaria que pueda recuperar el valor de la identidad personal, de la apropiación consciente de los fines de la propia vida y de la libertad como bien insustituible en la reciprocidad de las acciones humanas. Para ello no basta con la ayuda de la informática sino que están invitadas a sumarse la filosofía, las neurociencias, la psicología, la antropología y la sociología, entre otras. Es una larga lista que comparte el mismo enfoque formal: averiguar en qué aspectos las herramientas digitales están cambiando al hombre y a la sociedad, y con qué profundidad. Muchos entienden que esas innovaciones se darán (se están dando...) en primer lugar en las instituciones educativas. El libro de Cathy N. Davidson y David T. Goldberg titulado The future of thinking: learning institutions in a digital age (MIT Press, 2010) intenta evaluar la dimensión de esos procesos ligados con la aparición de "instituciones educativas virtuales" en las que la flexibilidad de su organización, la porosidad de sus fronteras y su potencialidad para explorar y originar nuevos productos culturales es asombrosa. Otra obra escrita por Mark Bauerlein, The dumbest generation: how the digital age stupefies Young americans and jeopardizes our future (or, don't trust anyone under 30) (Penguin Books, 2008) pone el acento en la aparición de una generación (las llamadas Y, Millennials o PuntoNet) que, a pesar de haber tenido acceso a una sociedad de abundancia (tanto intelectual como económica) en la que pudieron asistir a debates cívicos, consultar blogs políticos, leer libros antiguos (agotados) y hasta bibliotecas enteras on line, consultar canales de video sobre Historia o Biología con producciones adaptadas a los formatos actuales (en lugar de los clásicos documentales), y gozar de una actualización constante de la información -es decir, que tuvieron como nunca antes la oportunidad de educarse, aprender, actuar políticamente e involucrarse en la cultura- han cerrado su mente y han contraído su horizonte hasta los límites de su propio interés. "Cuanto más 
atienden sí mismos, menos se preocupan de recordar el pasado y proyectar el futuro" -declara Bauerlein (2008, p. 10). No han logrado más conocimiento, ni ser más inquisitivos, ni estar más "al día" con las noticias, de modo que sus capacidades digitales parecieran no permitirles mejorar el espíritu investigativo ni cumplir adecuadamente las tareas de sus lugares de trabajo. Este punto de vista sociológico, que considera la "generación" como la unidad de análisis la volvemos a encontrar en el libro de Howard Gardner y Katie Davis, The App generation: how today's youth navigate identity, intimacy and imagination in a digital world (Yale University Press, 2013), donde se reitera la transformación a la que nos referimos como un cambio generacional: estaría ingresando en escena una novedosa "Generación App" (por "aplicación"1, en inglés) de características bien definidas. La tesis defendida por Gardner es que los jóvenes piensan el mundo que los rodea como un ensamble de "apps" o, incluso, su propia vida como una sucesión ordenada de ellas -o una única "super-app" extendida que va desde la cuna hasta la tumba-. En este convencimiento subyace la idea de que todo lo que el hombre pudiera desear a lo largo de los años debería ser provisto por una "app" específica. Por eso, Gardner distingue entre personalidades "app-dependientes" (que restringen sus metas vitales y elecciones) y "appfacilitadoras" (que amplían las capacidades). Nuevamente aparece el riesgo de tomar el camino equivocado en esta era hyper-digital pero lo más importante resulta la evaluación de las modificaciones psicológicas y sociales a las que cualquiera de ambas alternativa conduce. La decantación de la rapidez y de la eficacia "justo a tiempo" propia de los dispositivos digitales se esparce, entonces, sobre cada región de la personalidad, construyendo ese tipo humano que Gardner y Davis intentan definir como miembro de la "Generación App". Por lo tanto, el papel que juega la educación en la detección y corrección del «estilo» vital que imprime la civilización digital en los jóvenes es fundamental para prevenir el desborde de las particularidades asociadas con la diagnosticada egomanía y aislamiento de los usuarios precoces de aquella tecnología. Se trata, pues, de avanzar en la ruta que nos conduzca a desentrañar cuál es la identidad (¿múltiple? ¿fluctuante?...) del nuevo sujeto digital y, consecuentemente, a establecer cuáles serían los requerimientos en la provisión de recursos para que sus aprendizajes fueran socialmente significativos y apropiados para remitirlos otra vez a la realidad, de la cual las tendencias a la virtualización y a los desbordes imaginarios parecieran alejarlos.

Conviene recordar que la situación se torna más urgente para todos aquellos que sólo han conocido un ambiente digital, precisamente a los que se refieren John Palfrey y Urs Gasser en Born Digital: understanding the first generation of digital natives (Basic Books, 2008) indicando que estos "nativos digitales" no perciben una dualidad en su identidad (la "identidad digital" y la "identidad real" -espacio-temporalmente determinada) sino que la conciben en una multiplicidad de representaciones de un único sujeto. Para ellos, las tecnologías digitales son los mediadores naturales para los contactos persona-a-persona, que pueden realizarse durante los siete días de la semana, 24 horas, sin restricciones geográficas. Uno de los pioneros en preocuparse por la educación de esos "nativos digitales" ha sido Marc Prensky en su obra Teaching digital natives. Partnering for real learning (Corwin, 2010)2 en la que sostiene que es necesario revisar las prácticas pedagógicas para dar lugar a la "coasociación" entendida como la distribución de responsabilidades en el proceso de aprendizaje siendo que corresponde a los estudiantes (solos o en grupos) buscar, hacer hipótesis, encontrar respuestas y crear presentaciones, y al profesor y la clase, valorar y examinar la corrección, contexto, rigor y calidad de aquellas intervenciones. Esta manera diferente de tratar el hecho educativo no sería posible sin contar con herramientas que posibiliten una personalización de los aprendizajes y en consecuencia, establecer los ritmos de estudio apropiados a cada estudiante. Al mismo tiempo, la misma tecnología que hace factible lo anterior conduce a la creación de una "sociedad" entre el profesor y el alumno y al intercambio de roles donde el "investigador" no necesariamente es el profesor -más bien al contrario- y el "crítico" el alumno -más bien al contrario. Bajo esta 
nueva forma, el alumno como "usuario experto" de la tecnología (pues ha nacido en un mundo mediatizado por ella) a través de pizarras interactivas, ordenadores portá- tiles, podcast, blogs, wikis o cualquier otro recurso resultará el verdadero protagonista del proceso educativo. El profesor se reserva el papel de orientador y guía, y quien puede ver "a largo plazo" las metas que se están persiguiendo. De ahí que, a criterio de Prensky, haya que consolidar una renovación curricular profunda para hacer relevante y eficaz lo que se aprende en las instituciones de enseñanza3. Pero si bien está claro que el entorno digital facilita el diálogo con el mundo circundante y transforma a la educación en un ámbito adaptativo para la sociedad del conocimiento, por otra parte, conviene considerar aspectos de lo humano que podrían estar perdiéndose o debilitándose en dicha transición. Quizás uno de los alegatos más contundentes en ese sentido lo encontremos en la obra de Raffaele Simone titulada La Terza Fase. Forme di sapere che stiamo perdendo (Laterza, 2000)4 . Allí el autor presenta un panorama de la evolución en las formas de adquisición del conocimiento:

Se trata de lo siguiente: a finales del siglo xx hemos pasado gradualmente de un estado en el que el conocimiento evolucionado se adquiría sobre todo a través del libro y la escritura (es decir, a través del ojo y la visión alfabética o, si se prefiere, a través de la inteligencia secuencial), a un estado en el que éste se adquiere también -y para algunos principalmente- a través de la escucha (es decir, el oído) o la visión no-alfabética (que es una modalidad específica del ojo), es decir, a través de la inteligencia simultá- nea. Hemos pasado, así pues, de una modalidad de conocimiento en la cual prevalecía la linealidad a otra en la que prevalece la simultaneidad de los estímulos y de la elaboración (2001, p. 37).

Simone asocia el alfabetismo con ciertas formas de inteligencia que se desprenderían de él y que han constituido la base del desarrollo occidental en los siglos posteriores a Gutemberg. Por eso afirma, con cierto pesimismo que "estamos retornando al dominio del oído y de la visión no alfabética, y las jóvenes generaciones son una vanguardia de esta migración de retroceso" (Simone, 2001, p. 39). En síntesis, la que llamaríamos "inteligencia alfabética" significó la adquisición de habilidades mentales que hasta entonces no eran funcionales, por ejemplo, el ordenamiento y clasificación de datos e informaciones generando útiles como los ficheros, índices, repertorios, etc.; se crearon imágenes impresas con fines explicativos que facilitaron los desarrollos tecnológicos, y se abandonaron ayudas mnemotécnicas por la presencialidad del texto. Ahora bien, lo más interesante que señala, quizás, sea el hecho de que esa modificación de los hábitos intelectuales conllevó una alteración de las formas de sociabilidad. El pasar de una "sociedad de oyentes" -por tanto, acostumbrada a la compañía- a una "sociedad de lectores" -más individual- implicó un cambio revolucionario en el significado de "estar juntos". A esa descripción de la inteligencia gutembergiana y sus consecuencias, le seguiría una nueva etapa -la "tercera fase" del título- caracterizada por una falta de capacidad del ojo para seguir la escritura, hasta entonces la principal fuente de conocimiento, pues han crecido y se han instalado otras opciones igualmente ricas de información accesibles al oído y a la visión no-alfabética. La conclusión de Simone es que el hombre contemporáneo "ha renunciado así a una conquista evolutiva que había sido estimulada por la escritura, para dar un paso atrás. Es casi como si se dejase de lado la visión alfabética -un medium lleno de tensiones y "fatigas"- para volver a unos media más naturales, más primitivos, con menor grado de gobierno" (Simone, 2001, p. 43). La hipótesis de Simone es ciertamente perturbadora, pero se anima a establecer que la presencia de nuevas tecnologías no es neutra al momento de constatar un uso intensivo, en especial, si su recepción se produce de manera temprana en la vida del usuario. Esto nos lleva al punto que queríamos subrayar: ya nos encontramos frente a un nuevo sujeto en sus múltiples dinámicas de interacción (como ciudadano, como miembro de una familia, como profesional, como integrante de una sociedad -particular o global-, etc.), un 
"sujeto digital" cuyos esquemas de percepción y pensamiento no son similares a los pasados. Se trata exagerando- de una "novedad antropológica" que habrá que comprender, analizar, guiar y, desde ya, educar.

\section{Vida social y mente digital}

Cuando Sherry Turkle señala la contradicción interna que detecta en la época de las comunicaciones, denuncia -al mismo tiempo- la falta de visión para construir relaciones estables y significativas con nuestro prójimo "a pesar de la tecnología". Su descripción presenta en forma concisa pero demoledora las consecuencias sociales de los hábitos que producen los dispositivos informáticos:

La tecnología promete dejarnos hacer cualquier cosa desde cualquier lugar con cualquier persona. Pero también nos agota ya que tratamos de hacer todo en todas partes. Empezamos a sentirnos abrumados y empobrecidos por la vida que la tecnología hace posible. Podemos tener la libertad de trabajar desde cualquier lugar, pero también somos propensos a estar solos en todas partes. En un giro sorprendente, la interconexión despiadada conduce a una nueva soledad. Nos dirigimos a las nuevas tecnologías para llenar el vacío, pero mientras la tecnología es una rampa que sube, nuestra vida emocional es una rampa que baja5 .

Falta, pues, el balance entre los beneficios utópicos de una era de la conexión total y la preparación anímica adecuada para responder a los desafíos de las relaciones interpersonales mediatizadas ahora por las pantallas de las computadoras y los dispositivos móviles. Como sostiene John Seely, "contrariamente a las suposiciones de que todo lo que se necesita es la innovación tecnológica, en una revolución digital también se necesitan innovaciones no tecnológicas similares para concretar su potencial" (Seely, 2000, p. XXI). En este grupo habría que ubicar a las habilidades sociales -y no sólo a las intelectuales- que reclama este nuevo escenario digital. El peligro de separar el texto del contexto, y metafóricamente, de no saber integrar el texto digital con las conductas contextuales que se desprenden de aquél, lleva a ignorar la profunda implicación que existe entre ciertos procesos sociales que sirven a las plataformas de eficiencia tecnológica y la lógica de la digitalización global. En pocas palabras, la modificación de las prácticas cognitivas que supone el empleo severo y constante de dispositivos y redes digitales, se traduce en cambios ya apreciables en los comportamientos de sus usuarios. Algunos hablan de una visión "infocéntrica" que deja en la periferia a esas repercusiones sociales e incluso se niegan a considerar que sin los aportes de las organizaciones involucradas en el desarrollo tecnológico (universidades, industrias, grupos de investigación, etc.) no sería posible la generación del conocimiento y su disponibilidad. Ortega y Gasset, filósofo español con reputación mundial por su libro La Rebelión de las Masas, apuntaba en esa obra que la «naturalización» de la técnica (que, en rigor, había sido obra de un enorme esfuerzo realizado por grandes porciones de la humanidad) llevaría a olvidar que sin las condiciones adecuadas para desarrollar la teoría -representada por la filosofía y la ciencia- volveríamos a una edad primitiva, incapaces de vincularnos con el mundo de una manera creativa para asegurar la vida del hombre. Pensar que el entorno tecnológico está allí simplemente para su disfrute sin medir las proporciones de la intervención humana para lograrlo es anticipar el ocaso de su continuación. Por lo tanto, hay que evitar la ingenuidad de concebir una civilización hiperconectada sin un pasado de gestación compleja y sin consecuencias para el hombre que participa de ella. Una "vida digital" no se reduce a la ejecución de apps y programas en días y horarios establecidos, en una duplicidad entre "lo que soy" y "lo que hago" (separación imposible dada la inmersión casi total que exige la sociedad del siglo XXI) sino que solicita un determinado perfil para que la eficacia del instrumento alcance el máximo rendimiento, y que se extiende sobre la totalidad de la existencia. En este punto resulta sintomática la apelación reiterada a que, gracias a Internet, el futuro del trabajo estará entre las paredes 
del hogar. La home office se presentaba como el ideal de entorno laboral pues garantizaba libertad de horarios, flexibilidad para atender otros deberes (niños, mascotas, etc.) y alto rendimiento. La fuerte demanda de independencia estaría satisfecha y el trabajador podría cumplir sus tareas desde cualquier lugar con sólo contar con una laptop y un celular: un verdadero habitante de la comunidad global sin límites físicos ni fronteras políticas. Sin embargo, la realidad de los últimos años nos acercó un escenario bien distinto: la tecnología que administra un trabajador aislado es falible, debe enfrentar virus y corrupción de sus datos, mensajes que no llegan, incompatibilidades, y otros problemas asociados con hardware o software defectuosos. La necesidad de contar con la ayuda de otros (para solucionar problemas o para aunar criterios) no desaparece y las interacciones se convierten en imprescindibles. De hecho, se ha dado recientemente una reversión en la dirección hacia el home working y se retorna a un ámbito laboral compartido. Si bien, en este caso, se organizan las actividades para que no se multiplique el soporte en papel y los puestos de trabajo se intercambian, incluso diariamente, para que nadie pueda reclamar "su" lugar. Resulta evidente, entonces, que la vida digital se inserta en un sistema social de diversas escalas y hasta se podría decir que lo necesita. La colaboración es esencial pues no existe en cada casa un experto que pueda atender todas las consultas ni especialistas para los problemas recurrentes. La vuelta a la oficina convencional parece, por consiguiente, una tendencia que se afianzará con el paso del tiempo. La idea de que las tecnologías personalizadas (como las TIC's) aseguran un antídoto eficaz contra la masificación que produjo la revolución industrial no es una tesis que se sostenga hoy con facilidad y demuestra la complejidad que liga la vida digital con las condiciones sociales del medio en que se desarrolla. Pero si en el medio laboral la quimera del trabajo ubicuo y distribuido por redes globales ha recibido una crítica confrontativa, la idealización de los "ayudantes" digitales con características humanoides se mantiene firme: la robótica ha avanzado de manera considerable para ofrecer maquinarias capaces de cumplir tareas hogareñas, de mantener una conversación trivial o incluso se han probado prototipos que funcionan como acompa- ñantes de ancianos o niños. El deseo de socializar con artefactos ya incursionó en el cine con ejemplos tan distantes como el de 2001: Odisea del Espacio (Kubrik, 1968) o Blade Runner (Scott, 1982) hasta los más contemporáneos como Her (Jonze, 2013) o Ex-machina (Garland, 2015). Sin embargo, con el tiempo a nuestro favor, habremos de ocuparnos de estas curiosas relaciones más adelante, cuando dejen las pantallas y podamos admitir que forman parte de nuestra experiencia cotidiana... Por ahora, interesa más concentrarnos en averiguar cómo la "mente digital" renueva las condiciones de la interacción con los otros e imagina intercambios regulados por reglas hasta ahora impensadas. Paradójicamente, a medida que crecen las "conexiones" estamos menos dispuestos a las "conversaciones" -como nota Turkle- y a distinguir entre los compromisos efectivos de una relación vivida y la neutralidad aséptica de los contactos entre pantallas. Así, la soledad verdadera que antes significaba una cierta plenitud espiritual en la actualidad es difícilmente tolerada. La dimensión social de la vida, pues, ocupa una parte sustancial de nuestro tiempo y expresa una pulsión creciente por revelar y participar la intimidad de cada uno bajo múltiples formas: "cada plataforma social, servicio social (...) app social, se convierte en una pieza fundamental del nuevo mundo social mediático" (Keen, 2012, pp. 9-10). Las denominadas "redes sociales" como Facebook, Google+, Linkedin y Twitter han instalado una fórmula de recepción-acción que minimiza la reflexión al servicio de una urgencia que se derrama sobre el resto de las actividades humanas. Las nuevas prácticas digitales han incorporado una novedad psicológica que antes no se verificaba con cada ciclo de innovación tecnológica: para utilizar la expresión que emplea Tor Nørretranders en su libro The user illusion (1998) la "exformación" residente ha integrado una tensión que suprime la instancia de control y exige una respuesta casi inmediata para todos los intercambios sociales. Ortega diría que hemos regresado a una cierta "barbarie" en la que los dinamismos que amortiguaban los contactos sociales se han erosionado y sólo queda lugar para la conducta instantánea. La reacción inmediata en las redes sociales 
obedece a esa "exformación" que nos urge desde el subconsciente a devolver cada estímulo con una réplica similar. ¿Cómo preparar, entonces, a los nuevos "sujetos digitales" para que no se conviertan en los miembros de una sociedad masificada de segunda generación6?

\section{Hacia una educación post-digital}

Hasta ahora hemos pasado revista a las notas que, según muchos autores, son propias de una civilización digital que ha asociado la tecnología de las comunicaciones y el procesamiento de información a casi todas las actividades cotidianas. Sin embargo, el resultado no ha sido neutro o puramente superficial, sino que está cambiando la percepción e, incluso, los procesos mentales que se disparan a partir de la manipulación habitual de datos, registros o documentos. La exaltación de la visión simultánea en contra de la visión secuencial, propia de la lectura, ha significado el ingreso en un nivel de comprensión que no es acumulativo sino intuitivo. Lo que sucede, pues, con ese método -que puede implicar un menor tiempo de procesamiento- es que se abandona la correlación entre antecedente y consecuente y, por lo tanto, se debilita la capacidad argumentativa. Lo que trataremos de hacer a continuación será, por una parte, revelar los condicionamientos de la "mente digital" -que amenazan con crear nuevos desequilibrios y comportamientos no deseables- $y$, por otra, advertir de la necesidad de alterar la metodología de enseñanza-aprendizaje porque el ambiente digital determina cuáles son los objetos que se atienden y cuáles los que se descartan. Por ello, si existe una urgencia en resignificar el rol del docente para que pueda liderar la formación de los alumnos en tanto "sujetos digitales", poseedores de una visión del mundo diferente a la de las generaciones anteriores y con una inmersión nativa en la cultura tecnomediática, habrá que generar conciencia sobre estas incipientes habilidades meta-cognitivas. En este sentido, no se propone una deconstrucción preventiva del modelo de enseñanza sino advertir que la inclusión de las tecnologías sociales en los espacios educativos modificará -a largo plazo- los patrones y esquemas mentales con los que se administra la información. Frente a este reconocimiento, la tarea pedagógica no puede consistir simplemente en un entrenamiento sobre dispositivos -los cuales, por otra parte, estarán en continua evolución y cambio- sino en la generación de los resortes internos que exploren, filtren y-ocasionalmente- modifiquen los hábitos de apropiación de las competencias que surgen de las interacciones digitales. En última instancia, lo que se busca es contribuir a que el autoconocimiento o identidad del sujeto real no reciba la interferencia de la subjetividad digital. Por eso, el trabajo docente debe tender progresivamente a la interdisciplinariedad en el ecosistema del aula en orden a explorar todas las alternativas que admite la introducción del mundo digital en el campo educativo. Tengamos presente que hoy el acceso a muchas fuentes de información actualizada sólo se consigue a través de la conexión a bases masivas de datos por vía de buscadores (por ejemplo, Google), o la lectura de textos escaneados de libros agotados o antiguos no se puede efectuar sin un medio digital, o la realización de grabaciones audiovisuales como metodología de trabajo escolar que requieren para su posterior difusión de las redes sociales -con la sencilla ayuda de un smart-phone (teléfono inteligente) en lugar de una cámara de video-, sin mencionar las que se denominan "tecnologías conversacionales" (Facebook, Twitter, Instagram, etc.) que facilitan la creación de grupos de discusión o invitan a la participación instantánea con opiniones o incluso, encuestas rápidas. La virtualización educativa constituye, también, uno de los caminos de la transición cultural que cada vez más suma más partidarios; así, la autonomía se refuerza en detrimento de una práctica más colaborativa y horizontal. La construcción de blogs y de wikis que -a priori- puede suponer una mayor voluntad de compartir corre el riesgo de terminar en el desarrollo de una psicología autista en la que la exposición en la red no trasciende hacia un verdadero intercambio crítico. El paradigma de una "cibereducación" autosuficiente y promovida por la sociedad abierta en relación directa con los ciudadanos que la forman, 
rivalizando como alternativa a la escuela, dependiente del reconocimiento explícito de su lugar educativo y de su vertebración a partir del concepto de autoridad, plantea una falsa disyuntiva pero atrae a muchos dispuestos a banalizar la influencia de la tecnología. Con lo dicho, empieza a verse claramente que la oposición entre un paradigma de educación informal (sería la que habilita la virtualidad y omnipresencia de recursos informativos e instruccionales -por ejemplo, los "tutoriales" que se pueden consultar en YouTube- distribuidos por la sociedad) y otro paradigma de educación formal (por someterse a un encuadre institucional -la escuela, la universidad) es inconsistente, y no tiene en cuenta que en aquella "educación informal" ya existe una formalidad (quizás más exigente que la escolar) que se asume desde la inconsciencia del rigor preceptivo de ciertas aptitudes que el sistema requiere para "funcionar" y para garantizar que cada ser humano sea partícipe activo de los beneficios que promete. Hay una variación muy sutil entre la apelación que se realiza a las "tecnologías de la información y la comunicación" (TIC's), y las "tecnologías sociales" que parecen construir y regular los puentes entre las personas, y entre las personas y las organizaciones. No hablamos, pues, de una mera realidad instrumental sino de un factor identitario, que se incorpora a la misma trama de las relaciones entre el hombre y el mundo e influye sobre el perfil que define al proceso socializador. Sin duda, el lugar de la corporeidad como "medio" expresivo se torna aquí problemático en una trama de "sujetos digitales" y eso también significa una preocupación inédita para la formación de los alumnos. La creación de un "entorno digital" (como el de los videojuegos, pero con intención educativa) desafía la flexibilidad de las categorías de instalación somática y abre posibilidades indefinidas para la autoconstrucción del yo. Al respecto, esa facilidad remite a una multiplicidad de egos virtuales, digitales- cuya vertebración será difícil de conseguir de no mediar una expresa apelación a su control gracias al proceso educativo. Ahora bien, el desarrollo de un nuevo orden simbólico asociado a la tecnología digital admite interrelacionar con pantallas en las que no siempre tengo la seguridad de que se alojen subjetividades humanas como contraparte de mis acciones. En esos intercambios se dinamizan emociones y sentimientos, lo cual vuelve a crear un interrogante superlativo: ¿cuáles son las implicaciones antropológicas de estas herramientas? El tratamiento de los "otros reales" como "otros digitales" plantea una curiosa inversión del test de Turing porque las expectativas de que el ser humano asuma respuestas de computadora (rapidez, precisión, etc.) es cada vez más usual y menos sorpresivo. Este proceso de transferencia -sin otorgarle un sentido psicoanalítico- no se reduce a características subordinadas o secundarias sino que se activa en el lenguaje y en la capacidad de representación del mundo. Si antes primaba el orden verbal lineal, ahora, la percepción reticular y la hipertextualidad han producido un cambio profundo en nuestro sistema conceptual. Como lo advierte Landow (1995, p. 143), se han sustituido las ideas de "centro, margen, jerarquía y linealidad" por las de "multilinealidad, nudo, conexión y red". Podría decirse, al modo de Umberto Eco, que se ha desarrollado o construido un "usuario modelo" que incorpora a su subjetividad una visión pragmática y exploratoria similar a la que el sujeto digital emplea en la interacción con juegos o entretenimientos (gamificación). Estas nuevas competencias aprendidas se vuelcan luego en la manipulación textual y en la interpretación de situaciones o contextos de la vida. El caso de la "simulación" como soporte mental para imaginar consecuencias y ajustar variables, se presenta como una clave hermenéutica para entender la realidad en un plano que no es ni meramente empírico ni meramente racional sino que responde a la multidimensionalidad a la que está habituado el usuario digital. Por lo tanto, las estrategias para la adquisición de conocimiento han variado de acuerdo con la necesidad de navegar en textos polisémicos y en infografías, lo cual supone haber incorporado competencias para decodificar textos y gráficos, formular hipótesis, superar fragmentaciones, o imaginar modelizaciones. Frente a tantos desafíos, la educación ha preferido ocuparse de enseñar a través de los medios (por ejemplo, la clase-invertida o flipped classroom, consiste en empoderar al alumno como protagonista de su aprendizaje gracias a los recursos tecnológicos) y no de analizar qué deja ese 
uso intenso y programado como sedimento en la personalidad de los estudiantes. Una nueva alfabetización digital no debería prescindir de un enfoque antropológico que pusiera de manifiesto la necesidad de alcanzar una etapa de post-digitalización. En ella, investigadores y profesores deberían estar dispuestos para elaborar un meta-análisis de la cibercultura que incluya las dimensiones educativa, psicológica y social. Ese que podríamos denominar "curriculum de intervención digital" sería un aporte a la comprensión del "sujeto digital" y a la búsqueda constante por adecuar el proceso de enseñanza-aprendizaje a la formación multidimensional, pero siempre humanista, que exige nuestra civilización posmoderna.

Notes

1. Una «aplicación» (app, en su abreviatura habitual) en informática es un programa que lleva a cabo una función directamente para un usuario. 2. Edición en español: Prensky, M. (2015) Enseñar a nativos digitales. Una propuesta pedagógica para la sociedad del conocimiento. Buenos Aires: Ediciones SM. 3. "Aunque los padres y educadores se esfuerzan en conseguir que los niños aprendan con los métodos antiguos, el combustible que les ofrecen hoy a los chicos (es decir, el curriculum y los materiales) está muy por detrás de lo que necesitan los chicos actuales" (Prensky, p. 23-24). 4. Edición en español: La Tercera Fase. Formas de saber que estamos perdiendo (Taurus: Madrid, 2001). 5. Sherry Turkle, Alone Together en http://www.alonetogetherbook.com/ 6. En La Rebelión de las Masas, José Ortega y Gasset, entendía que la masificación del siglo XX -primera generación- había sido causada, durante el siglo XIX, por dos técnicas: una social, la democracia, y otra cognitiva, la tecnología industrial.

\section{Bibliografía}

Bauerlein, M. (2008). The dumbest generation: how the digital age stupefies Young americans and jeopardizes our future (or, don't trust anyone under 30). Penguin Books. Buckingham, D. et al. (2005). The Media Literacy of Children and Young People: A Review of the Academic Research. London: Ofcom. Buckingham, D. (2006). "La educación para los medios en la era de la tecnología digital" - Ponencia para el Congreso del décimo aniversario de MED "La sapienza di comunicare”, Roma, 3-4 de Marzo 2006. Buckingham, D. y Willett, R. (eds.). "Is there a digital generation?" en Digital Generations: Children, Young People and New Media. Mahwah, NJ: Erlbaum. Burbules, N. y Callister, T. (h) (2001). Riesgos y promesas de las Nuevas Tecnologías de la Información. Buenos Aires: GRANICA - Educación. Canali, L. E. (2005). "El docente tutor on-line: la autoevaluación de las competencias básicas". Ponencia presentada en el Congreso Latinoamericano de Educación Superior en el Siglo XXI, Universidad Católica Argentina. Carbone, G. (1995). El lugar de las tecnologías en la Formación Docente. Presentación al II Congreso Iberoamericano de Educared "Educación y Nuevas Tecnologías", organizado por la Fundación Telefónica, Buenos Aires. Carr, N. G. (2010). The Shallows: What the Internet is doing to our brains, Norton \& Co. [Edición en español: Superficiales: ¿Que está haciendo Internet con nuestras mentes? Taurus, 2011] Davidson, C. N. y Goldberg, D. T. (2010). The future of thinking: learning institutions in a digital age. MIT Press. Dedé, Ch. (Comp.) (2000). Aprender con Tecnología. Buenos Aires: Paidós. De Las Heras, A. (1991). Navegar por la información. Madrid: Fundesco.

Eco, U. (1991). Lector in fabula. Barcelona: Lumen. Eisenstein, E. (1994). La revolución de la imprenta en la Edad Moderna europea. Madrid: Akal. Gardner, H. y Davis, K. (2013). The App generation: how today's youth navigate identity, intimacy and imagination in a digital world. Yale University Press. Gates, B. (1995). The road ahead [Edición en español: Camino al futuro]. Gee, J. (2003). What Video Games Have To Teach Us About 
Learning and Literacy. Basingstoke: Palgrave Macmillan. Johnson, S. (2005). Everything Bad is Good for You. Londres: Penguin Group. Keen, A. (2007). The Cult of the Amateur: How Today's Internet Is Killing Our Culture, Doubleday/Currency. Keen, A. (2012). Digital Vertigo. How Today's Online Social Revolution Is Dividing, Diminishing, and Disorienting Us. New York: St.Martins Press. Keen, A. (2015). The internet is not the answer. Grove/Atlantic. Landow, G. (1995). Hipertexto. La convergencia de la teoría crítica contemporánea y la tecnología. Barcelona: Paidós. Lanier, J. (2011). You are not a gadget. New York: Penguin/Random House. Leroi-Gourhan, A. (1971). El gesto y la palabra. Caracas: Publicaciones de la Universidad Central de Venezuela. Lévy, P. (1992). Le tecnologie dell'inteligenza. Boloña: Synergon. Litwin, E. (Comp.) (1995). Tecnología Educativa. Política, historias, propuestas. Buenos Aires: Paidós. Maldonado, T. (1998). Crítica de la Razón Informática. Barcelona: Paidós. Manovich, L. (2005). El lenguaje de los nuevos medios de comunicación. La imagen en la era digital. Barcelona: Paidós. Mcluhan, M. (1985). La Galaxia Gutenberg. Barcelona: Planeta-De Agostini. Morozov, E. (2011). The Net Delusion: The dark side of Internet freedom. Public Affairs. Negroponte, N. (1995). Being Digital [Edición en español: Ser Digital. Buenos Aires: Atlántida] Nørretranders, T. (1998). The user illusion. Ong, W. (1987). Oralidad y escritura. Tecnologías de la palabra. México: FCE. Ortega y Gasset, J. La Rebelión de las Masas. Papert, S. (1980). Mindstorms: Children, Computers and Powerful Ideas. New York: Basic Books. Postman, N. (1993). Technopoly: the surrender of culture to technology (Vintage Books). Rheingold, H. (1993). The virtual community. MIT PRess [Edición en español: Comunidades virtuales] Rodino, A. M. (1996). "Las nuevas tecnologías informáticas en la educación: viejos y nuevos desafíos para la reflexión pedagógica”. En Memoria del VII Congreso Internacional sobre Tecnología y Educación a Distancia. (pp. 51-71). Costa Rica: EUNED. Sartori, G. (1998). Homo videns. La sociedad teledirigida. Madrid: Taurus. Seely Brown, J. \& Duguid, P. (2000). The Social Life of Information. Harvard Business School Press [Edición en español: La vida social de la información. Prentice Hall, 2001] Shirky, C. (2010). Cognitive Surplus: Creativity and Generosity in a Connected Age. Penguin Press.

Scolari, C. (2008). Hipermediaciones. Elementos para una teoría de la comunicación digital interactiva. Barcelona: Gedisa. Scolari, C. (2006). "La estética post-hipertextual”, ponencia presentada en el Seminario Internacional "Las literaturas del texto al hipertexto/Literatures from text to hypertext". Madrid: Universidad Complutense de Madrid. Simone, R. (2000). La Terza Fase. Forme di sapere che stiamo perdendo. Roma: Laterza [Simone, R., La tercera fase. Formas de saber que estamos perdiendo. Madrid: Taurus]. Simone, R. (2012). Pressi nella rete -la mente ai tempi del web-. Roma: Garzanti Ed. Turkle, S. (1995). Life on Screen, Simon \& Schuster [Edición en español: La vida en la pantalla. Barcelona: Planeta, 2011] Turkle, S. (2012). Alone Together. Why we expect more from technology and less from each other. Basic Books. Virilio, P. (1997). El cibermundo, la política de lo peor. Madrid: Cátedra. Westera, W. (2013). The Digital Turn: How the Internet Transforms Our Existence. Bloomington: AuthorHouse. Zangara, M. A. (1998). "La incorporación de las nuevas tecnologías de la Información y la comunicación a los diseños curriculares. Algunos temas críticos”. Ponencia presentada en el IV Congreso RIBIE, Brasilia.

\section{Abstract:}

If we had to quickly trace the evolution of exchanges between information technology and education we should distinguish at least three phases: pioneering experiences, integration and manipulation of digital resources and virtualization. However, this process of appropriation has also been maturing and therefore motivated to reflect on their own teaching practices, pointing limits and possibilities. Today, we are witnessing the entry into another stage of that evolutionary path that could be termed as "post-digital". Here they are, then, issues that challenge 
not only the way to educate (comprehensive didactics of knowledge) but also interpret the formation of new cultural habits whose exercise does not recognize mediations or eliminates instances of control. Often it proposes a logic of "direct action" behind which a power that needs to be recognized and measured by its consequences is hidden. The magnitude of this change happened within the "knowledge society" that generates an educational alert requesting our attention on the nascent organization criteria "Curriculum digital intervention"; this is an intervention that has spread already from objects to people. Therefore, the relationship with the devices, platform selection, availability of information, the ubiquity of consultations, intervention and network management, and application development is the new digital environment that requires transdisciplinary approaches and a humanizing approach to avoid the danger of generating a mass of subjects adapted to the paradigms of the XXI century.

Key words:

digital - education - networks - TIC'S - knowledge - subject.

\section{Resumo:}

Se tivéssemos que delinear a evolução dos intercâmbios entre as tecnologias da informação e a educação deveríamos distinguir ao menos três fases: as experiências pioneiras, a integração e a instrumentação de recursos digitais e virtualização. Contudo, o processo de apropriação foi também de maduração, e, portanto, motivou uma reflexão sobre as próprias práticas docentes, apontando limites e possibilidades. Talvez hoje assistamos ao ingresso em outra etapa de aquela trajetória evolutiva que se poderia denominar como de pósdigitalização. Aqui aparecem, então, questões que desafiam não somente o modo de educar (contida na didática dos saberes) senão que também interpelam a formação de novos hábitos culturais cujo exercício não reconhece mediatizações, elimina instancias de controle e, muitas vezes, propõe lógicas de ação direta detrás das quais se oculta um poder que precisa ser reconhecido e medido por suas conseqüências. A magnitude desta mudança sucedida no interior da sociedade do conhecimento dispara um alerta educativo que precisa de nossa atenção sobre os critérios de organização do novo currículo de intervenção digital, uma intervenção alargada desde os objetos até as pessoas. Por tudo isso, a relação com os dispositivos, a seleção de plataformas, a disponibilidade da informação, a ubiqüidade das consultas, a intervenção e administração de redes, e o desenvolvimento de aplicações constituem o novo ambiente digital que precisa abordagens transdisciplinares e uma mirada humanizante para não sucumbir ao perigo de gerar uma massificação dos sujeitos adaptada aos paradigmas do século XXI.

Palavras chave:

digital - educação - redes TICs - conhecimento - sujeito.

Los nuevos aprendizajes del sujeto digital fue publicado de la página 107 a página121 en Cuadernos del Centro de Estudios de Diseño y Comunicación Nº 64 